\title{
Theory of magnetoelastic dissipation due to domain wall width oscillation
}

\author{
Y. Liu and P. Grütter ${ }^{\mathrm{a})}$ \\ Centre for the Physics of Materials, Department of Physics, McGill University, Montréal, Québec, \\ Canada H3A 2 T8.
}

(Received 12 September 1997; accepted for publication 19 February 1998)

\begin{abstract}
This article presents a general treatment of magnetic dissipation due to domain wall width oscillation via magnetostriction in magnetic samples. The domain wall width is modeled as a harmonic oscillator. The parameters governing this oscillator (effective mass, stiffness, damping coefficient and driving force) are derived and expressed in terms of intrinsic magnetic parameters of magnetic materials. The magnetostriction induced damping of wall width oscillations is frictional in nature. An external ac magnetic field serves as a driving force of the oscillator. It is found that the response to the driving force depends very much on the micromagnetic structures of the magnetic domain wall. Different micromagnetic structures lead to different magnetic dissipation for a given external field. Besides giving a quantitative microscopic explanation to magnetic dissipation data measured by magnetic dissipation force microscopy, this theory predicts two new phenomena: one is that there is a minimum driving force for the wall width to oscillate and the other is a new resonance phenomenon, domain wall width resonance. (C) 1998 American Institute of Physics. [S0021-8979(98)08510-7]
\end{abstract}

\section{INTRODUCTION}

Magnetic force microscopy (MFM) has been used by Grütter et al. ${ }^{1}$ to measure local magnetic dissipation in magnetic samples. This technique correlates locally the dissipation with micromagnetic structures in a magnetic sample. The highly localized alternating magnetic field created by an oscillating magnetic tip above the sample interacts with local micromagnetic structures. The resulting energy dissipation in the sample is measured simultaneously with the magnetic force gradient in an ac MFM by monitoring both the damping and the resonant frequency change of the vibrating cantilever. The lateral resolution for dissipation measurements is at least as good as in the case of force gradient measurements (better than $100 \mathrm{~nm}$ ). The magnetic force gradient is a result of the interaction of the tip magnetization with the sample stray field, while the dissipation signal comes from the tip field influence on the micromagnetic structures in the sample. The experimental dissipation signal is associated with domain wall positions and shows strong dependence on the micromagnetic structures of the domain walls. ${ }^{1}$

In a previous publication, ${ }^{2}$ we proposed a model to explain the observed dissipation. We suggested that the alternating tip field modulates the domain wall width, resulting in magnetoelastic emission of phonons. These phonons carry energy from the cantilever, leading to the image contrast at domain walls. Quantitative agreement with experiments was obtained.

The aim of this article is to continue the work in Ref. 2, giving a general theory of the wall width oscillations and the resulting dissipation. We consider the wall width oscillation as a damped, driven harmonic oscillator whose parameters are expressed in terms of basic magnetic parameters of mag-

${ }^{a)}$ Electronic mail: grutter@physics.mcgill.ca netic materials and micromagnetic wall structures. This general theory includes the results previously obtained ${ }^{2}$ and predicts two new phenomena: a minimum driving force to induce wall width oscillations and a wall width resonance.

\section{EQUATION OF MOTION AND ITS SOLUTIONS}

Very generally, a wall width oscillation, $w(t)$, may be treated as a simple harmonic oscillator described by an equation which should contain an inertia term $m \ddot{w}$, a stiffness force $\alpha(\Delta w)$, a damping term ${ }^{3}$ and an external alternating driving force term related to the external AC magnetic field ${ }^{4}$. Here $m$ is the effective mass of the domain wall for width oscillations, $\alpha$ is the wall stiffness of the domain wall for width oscillations, and $w$ is the wall width. In most damping problems, damping terms of viscous nature are considered (i.e., damping force is proportional to velocity). For magnetic damping problems due to domain wall movement, the damping terms of viscous nature include eddy current damping $^{3}$ and other damping mechanisms involving electronic and ionic rearrangements due to magnetization changes. ${ }^{5-8}$ Eddy current damping is present in width oscillations of domain walls but is very small. ${ }^{2}$ In the following, we only consider damping due to magnetoelasticity in magnetic samples. For viscous damping $(F \approx \Delta \dot{w})$, the energy change rate per unit area of domain wall, $\Delta \dot{E}$, is proportional to $(\Delta \dot{w})^{2}$. Damping of magnetoelastic origin, however, cannot be described by a viscous damping term, since the elastic energy change rate is proportional to the rate of change of the wall width, $\Delta \dot{w}$. This can easily be seen, as a change in domain wall width, $\Delta w$, causes magnetization direction changes at the wall location, leading to an elastic energy change in the sample via magnetostriction. The local elastic energy per unit area of domain wall created by $\Delta w$ is given by $\Delta E=\frac{1}{2} c \lambda^{2} \cdot \Delta w$, where $c$ is the Young's modulus and $\lambda$ is 
the magnetostriction coefficient of the magnetic material. The elastic energy change is thus proportional to $\Delta w$. This means that the damping force of magnetostrictive origin is a constant, independent of $\Delta \dot{w}$ or $\Delta w$, and can be written as $-\beta \dot{w} /|\dot{w}|$. Considering only a damping term due to the magnetoelastic effect, the equation governing the width oscillation of the wall is then

$$
m \ddot{w}+\beta \frac{\dot{w}}{|\dot{w}|}+\alpha\left(w-w_{0}\right)=F,
$$

where $w_{0}$ is the equilibrium width of the domain wall and

$$
F=F_{0} \exp (i 2 \pi f t)
$$

is the force trying to oscillate the wall width. The amplitude, $F_{0}$, of this force depends on the external ac magnetic field which is a function of the tip oscillation amplitude.

Before discussing the oscillator parameters $m, \beta$ and $\alpha$, we give the solution of Eq. (1).

Letting $w=w_{0}+w_{1} \exp (i(2 \pi f t+\delta))$, the solution of Eqs. (1) and (2) gives

$$
w_{1}=\frac{\sqrt{F_{0}^{2}-\beta^{2}}}{\alpha-(2 \pi f)^{2} m},
$$

from which the resonant frequency of the wall width oscillation is determined and given by

$$
f_{0}=\frac{1}{2 \pi} \sqrt{\frac{\alpha}{m}} .
$$

Since the damping force in Eq. (1) is a friction force with a constant amplitude $\beta$ resisting the change in the wall width, the energy dissipated in one oscillation cycle is $4 \beta w_{1}$. So the energy dissipation rate is given by

$$
P=4 f \beta w_{1}=4 f \beta \frac{\sqrt{F_{0}^{2}-\beta^{2}}}{\alpha-(2 \pi f)^{2} m}
$$

needed to let the wall width oscillate and hence to cause energy dissipation. This property is a consequence of the frictional nature of the damping force on the dynamic solution of Eq. (1). For the wall width to change, the driving force must be bigger than the friction force.

\section{PARAMETERS GOVERNING THE WALL WIDTH OSCILLATOR}

\section{A. Effective mass $m$}

We now consider the mass per unit area of domain wall. The existence of domain wall mass in the context of wall movements was first demonstrated by Döring. ${ }^{9}$ Döring showed that the moving wall differed in energy from the wall at rest by a term due to demagnetization effects. This effect is proportional to the square of the wall speed. He was thus able to identify this as a kinetic energy term, and defined the constant of proportionality as one-half the mass of the wall. A simplified treatment was given by Becker ${ }^{10}$ and a review was given by Kittel and $\mathrm{Galt}^{11}$ on the mass of domain walls.

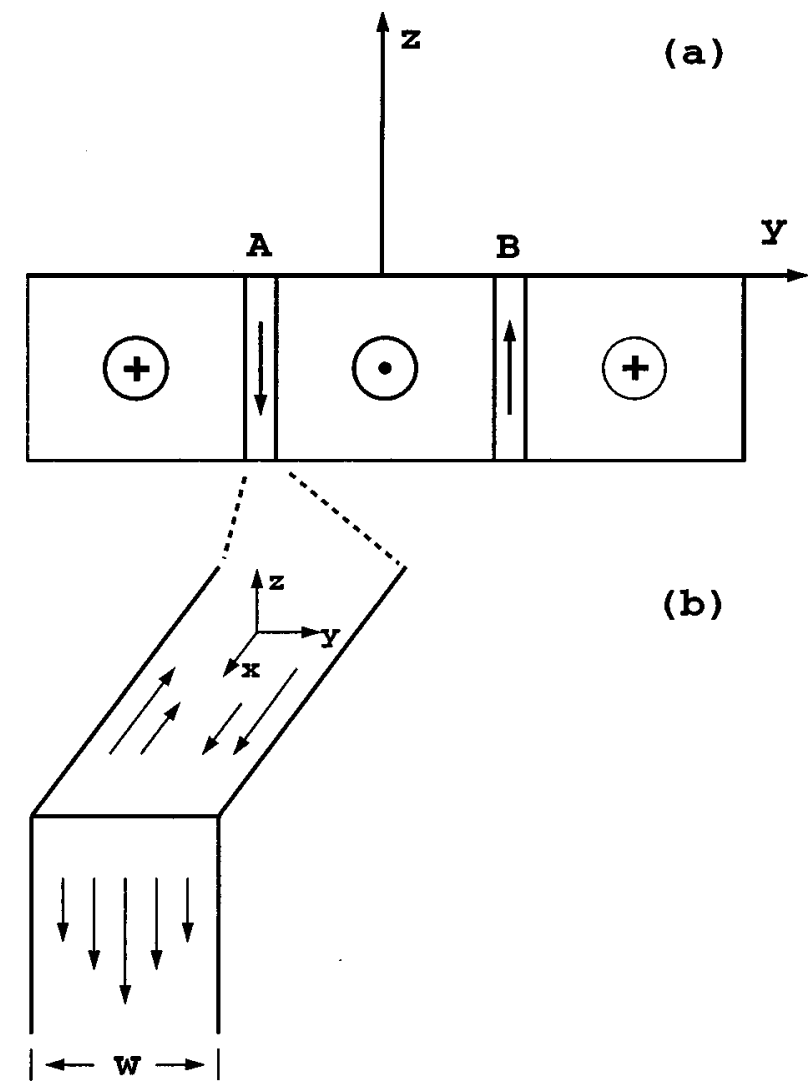

FIG. 1. (a) Magnetic domain structure in magnetic film with coordinate system, (b) $x$ and $z$ components of magnetization across domain wall A.

We now follow their basic physical idea to derive the effective mass of domain wall in the context of wall width oscillations. As an example, we consider a $180^{\circ}$ Bloch wall. Fig. 1(a) shows the magnetic domain structure with the coordinate system used. We choose the coordinate axes such that the $x z$ plane is the center plane of the wall. Let $\theta$ be the angle between the direction of the magnetization and the $z$ axis. Then $z$ axis is selected so that $\theta=90^{\circ}$ at one edge and $\theta=-90^{\circ}$ at the other edge of the domain wall. If the wall is at rest, the direction of the magnetization in the wall is always in the $x z$ plane perpendicular to the $y$ axis. If an external field is applied parallel to $z$, however, the magnetization in the wall tends to precess about the applied field. This causes the magnetization to turn in such a way that it has a component $M_{y}$ normal to the wall. Under these circumstances, a demagnetization field $H_{d}$ must be set up normal to the plane of the wall. The magnetization at any point inside the wall precesses around $H_{d}$ at the Larmor frequency. We assume that the angle between spins in adjacent atomic layers (along $y$ ) is constant in the wall [Fig.1(b)] even when the wall width is oscillating and, hence, $\theta=\pi y / w$ at point $y(|y|$ less than half the wall width $w$ ). The Larmor frequency is thus given by

$$
\dot{\theta}=-\pi \frac{y}{w^{2}} \dot{w} .
$$

The demagnetization field $H_{d}$ is given by the Larmor relation 


$$
H_{d}=\frac{1}{\gamma} \dot{\theta}
$$

where $\gamma=\left(\mu_{0} e g / 2 m_{e}\right)$ is the gyromagnetic ratio for the material; $g$ is the spectroscopic splitting factor and has a value between 1 and 2 ; $e\left(=1.6 \times 10^{-19} \mathrm{C}\right)$ is the electron charge and $m_{e}$ is the electron mass. From this demagnetization field, we can calculate the demagnetization energy per unit domain wall area. Note that this demagnetization energy is associated with changes in the wall width.

The effective mass for wall width oscillations per unit domain wall area may now be calculated by recognizing that since $H_{d}$ is proportional to $\dot{w}$, the demagnetization energy is proportional to $\dot{w}^{2}$, and is, therefore, the kinetic energy of the wall. Thus,

$$
\frac{1}{2} m \dot{w}^{2}=\frac{\mu_{0}}{8 \pi} \int H_{d}^{2} d V,
$$

where the integration is carried out over a cylinder of unit area normal to the wall and extends to infinity in both directions. The right side of this equation is a field energy not related to interactions within a material, thus the vacuum permeability $\mu_{0}$ is used instead of the material permeability $\mu$. From Eqs. (8), (7), and (6),

$$
m=\frac{\mu_{0}}{8 \pi} \frac{2}{\gamma^{2}}\left(\frac{\pi}{w_{0}^{2}}\right)^{2} \int_{-\frac{1}{2} w_{0}}^{\frac{1}{2} w_{0}} y^{2} d y=\frac{\pi \mu_{0}}{48 \gamma^{2} w_{0}} .
$$

The effective mass is inversely proportional to the equilibrium value of the wall width $w_{0}$. For Néel walls, Eq. (9) is still valid if one defines $\theta$ as the angle between the spins in the Néel wall and the $y$ axis. This means that the effective mass for the wall width oscillation does not depend on the wall micromagnetic structure as long as the assumed $\theta$ dependence on $y$ holds.

\section{B. Frictional damping coefficient $\boldsymbol{\beta}$}

As discussed in Sec. II, the damping force coefficient due to magnetoelastic effects is independent of the speed of the wall width change. The frictional damping force coefficient can be calculated by noting that the local elastic energy created by the change in the wall width per unit area of the domain wall is proportional to the change in the wall width $\Delta w$, and is, therefore, the work done to overcome the damping force. The local elastic energy per unit area of domain wall created by $\Delta w$ is given by

$$
\Delta E=\frac{1}{2} c \lambda^{2} \cdot \Delta w,
$$

where $c$ is the Young's modulus and $\lambda$ is the magnetostriction coefficient of the magnetic material. By setting $\Delta E=\beta$ - $\Delta w$, we obtain the frictional damping force coefficient $\beta$ per unit area of domain wall

$$
\beta=\frac{1}{2} c \lambda^{2}
$$

which is independent of the micromagnetic structures of domain walls.

\section{Wall stiffness $\alpha$}

The wall stiffness $\alpha$ per unit area of domain wall in the problems of wall width oscillation can be given by

$$
\alpha=\frac{\partial^{2} \sigma_{w}}{\partial w^{2}},
$$

where $\sigma_{w}$ is wall energy per unit area in the absence of an external field. At equilibrium width, the first order derivative of $\sigma_{w}$ with respect to $w$ is zero. For a wall width change $\Delta w$, the potential energy change of the domain wall is $(1 / 2) \alpha(\Delta w)^{2}$, from which it is easy to see that $\alpha$ represents the stiffness to wall width change. Ignoring the demagnetization energy in the wall, $\sigma_{w}$ contains exchange energy and anisotropy energy, ${ }^{2}$

$$
\sigma_{w}=\frac{J S^{2} \pi^{2}}{a} \cdot \frac{1}{w}+\frac{K_{1}}{2} \cdot w,
$$

where $J$ is the exchange constant between adjacent spins of angular momentum $\hbar S, K_{1}$ is the anisotropy constant, and $a$ is the unit cell dimension of the sample. A sample with uniaxial anisotropy is considered here. In deriving Eq. (13), it was assumed that the spins inside the wall rotate their direction with a constant angle between the adjacent spins. The wall stiffness $\alpha$ at the equilibrium width of the domain wall, $w_{0}$, is then

$$
\alpha=\frac{K_{1}}{w_{0}},
$$

where $w_{0}=\sqrt{2 J S^{2} \pi^{2} / K_{1} a}$ is the wall width without external field. This wall stiffness is valid for both Bloch and Néel walls. This is correct as long as the wall demagnetization energy can be neglected, resulting in an expression for the wall energy $\sigma_{w}$ as given by Eqs. (13). As discussed in Ref. 2, when the demagnetization energy in the wall is not negligible, the above equations are still valid if we replace the anisotropy constant, $K_{1}$, by an effective one, $K_{\text {eff }}$, with $K_{\text {eff }}>K_{1}$. In thin films with in-plane magnetization, the demagnetization energy for a Néel wall is negligible, while for a Bloch wall it is not negligible. Bloch walls thus have a higher stiffness $\alpha$.

\section{Driving force $\boldsymbol{F}$ for the wall width oscillation}

The driving force $F$ for wall width oscillation is a result of the modulated magnetostatic energy of the domain wall due to the external ac magnetic field. The magnetostatic energy strongly depends on the relative orientation of the external field with respect to the magnetization direction of the sample magnetic structure. A strong dependence of $F$ on the micromagnetic structures of the domain wall is expected for a given external field. An additional magnetostatic energy $\sigma_{\text {sta }}=\left[ \pm\left(2 \mu_{0} M_{s} H_{z} / \pi\right) \cdot w\right]$ is added to the domain wall when an external field $H_{z}$ along the $z$ direction is applied to a sample with the magnetization configuration shown in Fig. $1\left(180^{\circ}\right.$ Bloch wall). Here $(2 / \pi) M_{s}$ is the average magnetization $^{12}$ in the walls with $M_{s}$ being the saturation magnetization of the sample. The " + " sign is for A and the 


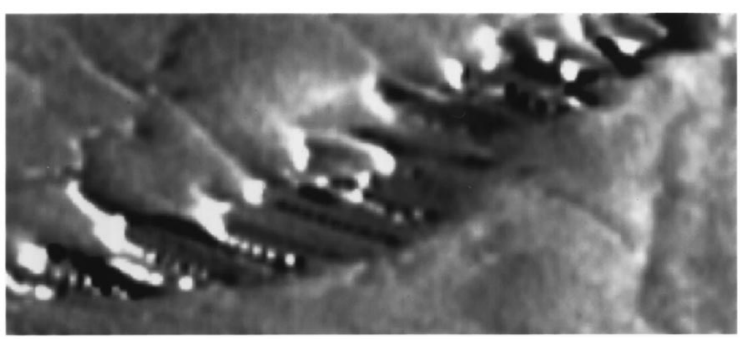

(a)

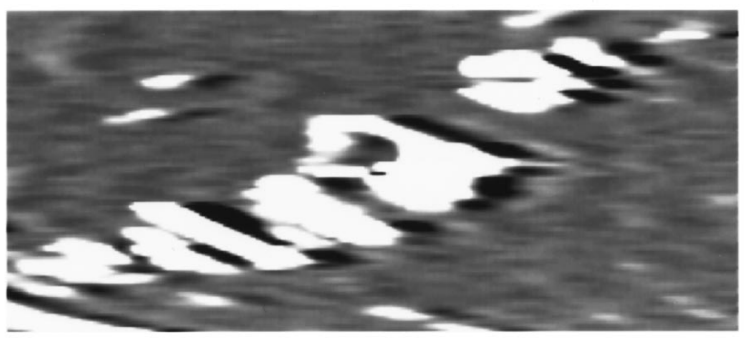

(b)

FIG. 2. MFM image and the simultaneously acquired magnetic dissipation image of a magnetic domain wall on a $30 \mathrm{~nm}$ thick Permalloy film. Image size: $8 \mu \mathrm{m} \times 3.5 \mu \mathrm{m}$. (a) Is the differentiated MFM image, (b) is the magnetic dissipation image.

“_,' sign is for wall B (Fig. 1). The driving force can be obtained from $F=-\left(\partial \sigma_{s t a} / \partial w\right)$, resulting in

$$
F= \pm \frac{2 \mu_{0} M_{s} H_{z}}{\pi}
$$

A field in the $x$ direction, $H_{x}$, has no effect on the wall width since it adds no energy to the walls. However, $H_{x}$ exerts a force leading to wall position displacements as it adds magnetostatic energies of opposite signs to the adjacent magnetic domains. We won't consider dissipation due to wall displacement in this article although every tip field has both $H_{z}$ and $H_{x}$ components. A field in the $y$ direction, $H_{y}$, has no effect either on wall width or wall position for the $180^{\circ}$ Bloch walls.

For Néel walls, the average magnetization within the walls lies in the film plane, either parallel or antiparallel to the $y$ axis. $H_{x}$ and $H_{z}$ thus do not modulate the wall width. In this case $H_{z}$ in Eq. 15 should be replaced by $H_{y}$.

The fact that different micromagnetic structures of domain walls are sensitive to fields with different orientations provides us with a method to study micromagnetic structures of domain wall by measuring magnetic dissipation with a suitably magnetized tip. This has been experimentally confirmed. ${ }^{12}$ Figure 2 shows a magnetic dissipation image together with the simultaneously acquired MFM image on a segment of a domain wall in a $30 \mathrm{~nm}$ thick Permalloy film. The MFM domain wall image shows a number of micromagnetic structures which lead to variable contrast in the dissipation signal.

\section{NUMERICAL RESULTS AND DISCUSSIONS}

We have correlated $m, \beta$ and $\alpha$ with the magnetic parameters of materials and the equilibrium width of domain walls. As a numerical example we take Co: inserting the bulk cobalt values of $K_{1}\left(4.12 \times 10^{5} \mathrm{~J} / \mathrm{m}^{3}\right), J(1 \mathrm{eV}), a(2.5$ $\left.\times 10^{-10} \mathrm{~m}\right), M_{s}\left(1.4 \times 10^{6} \mathrm{~A} / \mathrm{m}\right), c\left(3.0 \times 10^{11} \mathrm{~N} / \mathrm{m}^{3}\right)$ and $\lambda$ $\left(-6 \times 10^{-5}\right)$ into $w_{0}$ and Eqs. (9), (11) and (14), we get $w_{0}$ $=175 \mathrm{~nm}, \quad m=1.1 \times 10^{-11} \mathrm{~kg} / \mathrm{m}^{2}, \beta=540 \mathrm{~N} / \mathrm{m}^{2}$ and $\alpha$ $=2.35 \times 10^{12}(\mathrm{~N} / \mathrm{m}) / \mathrm{m}^{2}$. Inserting these parameters into Eqs. (3) and (5), we find that the effect of the effective mass on the wall width oscillation and the associated dissipation is negligible if the frequency of the driving force is much less than $7.3 \times 10^{10} \mathrm{~Hz}$, the resonant frequency of the wall width. This condition is routinely met for dissipation measurements by MFM since the currently available highest cantilever frequency is only a few MHz.

In an ac MFM, the tip field can be written as $H=H_{0}$ $+H_{1} \cos (2 \pi f t)$ with $f$ being the oscillation frequency of the cantilever. The dc part, $H_{0}$, exerts a constant force on the wall width and so causes a change in equilibrium wall width, while the ac part, $H_{1} \cos (2 \pi f t)$, causes the wall width to oscillate. The measured dissipation directly correlates with the ac part of the tip field. The effect of the dc tip field on the dissipation is a second order effect as it only influences the dissipation through the equilibrium value of wall width.

If $F_{0}>>\beta$, Eq. (5) can be rewritten as

$$
P=2 f c \lambda^{2} w_{0} \frac{2 \mu_{0} M_{s}}{K_{1} \pi} H_{z 1},
$$

which is basically identical to Eq. (3) of Ref. 2 except for a factor of 2. This difference comes from our different assumptions. In obtaining Eq. 3 of Ref. 2, we assumed that the dissipated energy in one cycle equals the elastic energy change when the wall width goes from its minimum value to its maximum value, while here we calculate the elastic energy change when the wall width goes from its minimum through maximum and back to its minimum width. Equation (16) is for Bloch walls. For Néel walls, $H_{z 1}$ should be replaced by $H_{y 1}$, where $H_{z 1}$ and $H_{y 1}$ are the amplitudes of the $z$ and $y$ components of the ac tip field, respectively. The condition that $F_{0}$ be much greater than $\beta\left(540 \mathrm{~N} / \mathrm{m}^{2}\right)$ leads to the requirement that $H_{z 1}$ or $H_{y 1}$ be much bigger than 6.05 Oe for a Co sample. The amplitude and distribution of the tip field depend on the tip magnetization and tip geometry. ${ }^{2,12}$ According to our field calculations, ${ }^{2,12}$ the condition is satisfied when a $90 \mathrm{~nm} \mathrm{CoPtCr}$ film coated $\mathrm{Si}_{3} \mathrm{~N}_{4}$ cantilever is used. Indeed, with a $90 \mathrm{~nm}$ CoPtCr film coated $\mathrm{Si}_{3} \mathrm{~N}_{4}$ tip, we experimentally observed a magnetic dissipation signal on a 4 $\mathrm{nm}$ Co film, which has been quantitatively explained by the magnetoelastic losses., ${ }^{2,12}$

For a $20 \mathrm{~nm}$ NiCo film coated Si cantilever, the condition $\left(H_{y 1}>6 \mathrm{Oe}\right)$ for wall width oscillations is not satisfied. With a $20 \mathrm{~nm}$ NiCo film coated Si tip, we do not observe any dissipation contrast in the Co film. For the $4 \mathrm{~nm}$ Co film, the domain wall is Néel-type and the in-plane tip field, $H_{y 1}$, is relevant for the dissipation.

The quadratic dependence of dissipation on the magnetostriction coefficient has been qualitatively confirmed by imaging a thin film Terfenol-D sample and a thin film NiFe sample with the same tip. ${ }^{1,12}$ Terfenol-D has a magnetostriction coefficient ${ }^{13}$ about 100 times larger than NiFe. We measured a corresponding dissipation increase in the Terfenol-D sample. 
The resonant frequency of the wall oscillation is given by Eq. (4). Putting in the expressions for $m$ and $\alpha$, we get $f_{0}=(\gamma / 2 \pi) \sqrt{48 K_{1} / \pi \mu_{0}}$ which is only dependent on the basic magnetic sample parameters, $\gamma$ and $K_{1}$. For most magnetic materials, due to the orbital momentum quenching, $\gamma$ is a constant. This leads to $f_{0}$ only depending on the anisotropy constant $K_{1}$ (or $K_{\text {eff }}$ if the demagnetization energy of the domain walls is not negligible). For Co samples, we get $f_{0}$ $=7.3 \times 10^{10} \mathrm{~Hz}$. This frequency value falls into a frequency range where a previously unexplained resonance peak in magnetic spectrum was observed in a Ferramic sample. ${ }^{14}$ This unexplained peak is the third resonance peak in the magnetic spectrum. The first two peaks were explained as results of domain wall position resonance and ferromagnetic resonance of magnetic domains in their internal field, respectively.

Note that the parameters governing the oscillation of the domain wall width are dependent only of the basic magnetic parameters of magnetic materials. This differs from the oscillation of the wall position in which the strength of pinning sites will contribute to the wall stiffness and, hence, the resonant properties.

\section{CONCLUSION}

In summary, we have proposed a general theory of magnetic domain wall width oscillations and the associated dissipation. Theoretical predictions on the dissipation are consistent with recent experiments. Due to the frictional nature of the damping force, a minimum driving force (equal to or bigger than the friction force) is required to cause wall width oscillations. The parameters governing wall width oscilla- tions are only dependent on intrinsic magnetic parameters of the material. For a given external field, the amplitude and the dissipation of the wall width oscillators depend on the relative orientation of the wall internal magnetization with respect to the external field. Resonances in domain wall width are predicted, which might be detected by methods of magnetic resonance force microscopy. ${ }^{15}$

\section{ACKNOWLEDGMENTS}

This work was supported by grants from the National Science and Engineering Research Council of Canada and Le Fonds pour la Formation des Chercheurs et l'Aide à la Recherche de la Province de Québec.

${ }^{1}$ P. Grütter, Y. Liu, P. LeBlanc, and U. Dürig, Appl. Phys. Lett. 71, 279 (1997).

${ }^{2}$ Y. Liu, B. Ellman, and P. Grütter, Appl. Phys. Lett. 71, 1418 (1997).

${ }^{3}$ H. J. Williams, W. Shockley, and C. Kittle, Phys. Rev. 80, 1090 (1950).

${ }^{4}$ In Ref. 2, we did not consider the effect of damping on the amplitude of the wall width oscillation.

${ }^{5}$ J. K. Galt, Bell Syst. Tech. J. 33, 1023 (1954).

${ }^{6}$ A. M. Clogston, Bell Syst. Tech. J. 34, 739 (1955).

${ }^{7}$ L. Néel, J. Phys. Radium 12, 339 (1951).

${ }^{8}$ L. Néel, J. Phys. Radium 13, 249 (1952).

${ }^{9}$ W. Döring, Z. Naturforsch. A 3a, 373 (1948).

${ }^{10}$ R. Becker, J. Phys. Radium 12, 332 (1951).

${ }^{11}$ C. Kittel and J. K. Galt, in Solid State Physics, edited by F. Seitz and D. Turnbull (Academic, New York, 1956), Vol. 3, p. 437.

${ }^{12}$ Y. Liu, Ph.D. thesis, McGill University, 1997; Y. Liu and P. Grütter (unpublished)

${ }^{13}$ Q. Su, Y. Zheng, A. Roytburd, and M. Wuttig, Appl. Phys. Lett. 66, 2424 (1995).

${ }^{14}$ G. T. Rado, R. W. Wright, and W. H. Emerson, Phys. Rev. 80, 273 (1950).

${ }^{15}$ J. A. Sidles, J. L. Garbini, K. J. Bruland, D. Rugar, O. Züger, S. Hoen, and C. S. Yannoni, Rev. Mod. Phys. 67, 249 (1995). 